\title{
MicroRNA-543 inhibits proliferation, invasion and induces apoptosis of glioblastoma cells by directly targeting ADAM9
}

\author{
TAO JI ${ }^{1}$, XIEJUN ZHANG ${ }^{1,2}$ and WEIPING $\mathrm{LI}^{1,2}$ \\ ${ }^{1}$ Department of Neurosurgery, Shenzhen Second People's Hospital, Clinical College of Anhui Medical University; \\ ${ }^{2}$ Shenzhen Key Laboratory of Neurosurgery, Shenzhen, Guangdong 518035, P.R. China
}

Received February 22, 2017; Accepted July 20, 2017

DOI: $10.3892 / \mathrm{mmr} .2017 .7332$

\begin{abstract}
Glioma is the most common type of malignant brain tumor in humans and accounts for $81 \%$ of all malignant brain tumor cases in adults. The abnormal expression of microRNAs (miRs) has been reported to be important in the formation and progression of various types of human cancer, including glioblastoma (GBM). Therefore, studies into the expression, and roles of microRNAs as diagnostic and prognostic markers, as well as their therapeutic value for patients with GBM are warranted. The expression and roles of miR-543 have been reported in several types of human cancer. However, the role of miR-543 in GBM remains unclear. In the current study, the expression pattern of miR-543 in GBM, the effects of miR-543 on GBM cells and the underlying molecular mechanism was determined. The results of the present study demonstrated that miR-543 was significantly downregulated in GBM tissue samples and cell lines. Furthermore, the upregulation of miR-543 inhibited GBM cell proliferation and invasion, as well as promoted cell apoptosis. In addition, a disintegrin and metalloproteinase 9 (ADAM9) was identified to be a direct target gene of miR-543. Furthermore, ADAM9 was significantly upregulated in GBM tissue samples and its expression was inversely correlated with miR-543 expression in GBM tissue, suggesting that miR-543 downregulation may contribute to ADAM9 upregulation in GBM. Finally, the results of the rescue experiment indicated that ADAM9 overexpression significantly reversed the effects of miR-543 on the proliferation, invasion and apoptosis of GBM cells, suggesting that miR-543 serves as a tumor suppressor in GBM through ADAM9 regulation. Overall, these findings indicate that the miR-543/ADAM9 signaling pathway may provide as a potential therapeutic strategy for GBM.
\end{abstract}

Correspondence to: Professor Weiping Li, Department of Neurosurgery, Shenzhen Second People's Hospital, Clinical College of Anhui Medical University, 3002 Sungang West Road, Shenzhen, Guangdong 518035, P.R. China

E-mail: liweiping0755@163.com

Key words: microRNA-543, glioblastoma, proliferation, invasion, apoptosis, a disintegrin and metalloproteinase 9

\section{Introduction}

Glioma is the most common type of malignant tumors in the brain; it originates from neural stromal cells and accounts for $81 \%$ of all malignant brain tumors in adults $(1,2)$. The formation and progression of glioma is extremely complex and involves aberrant activation of proto-oncogenes and inactivation of tumor suppressor genes (3-5). Glioma could be graded from grade I to grade IV, based on the degree of malignancy according to the World Health Organization (WHO) classification (6). Glioblastoma (GBM), the highest-grade glioma, is characterized by a heterogeneous population of cells that are genetically unstable, highly infiltrative, angiogenic, and resistant to chemotherapy (7). Despite recent advances in multimodal therapies, the prognosis of GBM patients remains poor, with an average five-year survival rate of only 4 to $5 \%(8,9)$. The average survival time of GBM remains only 14.6 months following surgical treatment combined with radiation and chemotherapy (10). The high mortality of GBM is partially due to rapid growth, angiogenesis and metastasis of GBM cells $(11,12)$. Therefore, improving understanding regarding the underlying mechanisms involved in GBM occurrence and progression, which would be essential to identify novel therapeutic methods for patients with this disease, is clinically significant.

MicroRNAs (miRNAs) are a group of endogenous, single-stranded and non-protein-coding short RNAs with approximately 19-24 nucleotides in length (13). miRNAs negatively regulate the gene expression through imperfect base pairing with the 3 -untranslated regions (3'-UTRs) of target messenger RNAs (mRNAs), resulting in translation repression or mRNA degradation (14). Each miRNA could directly modulate hundreds of target genes, and more than one-third of genes may be regulated by miRNAs (15). miRNAs play important roles in diverse biological processes, such as cell proliferation, cycle, death, differentiation, metastasis and drug resistance (16-18). The deregulation of miRNAs has been detected in a wide variety of human cancers, such as glioma (19), prostate cancer (20), gastric cancer (21), breast cancer (22) and lung cancer (23). Increasing evidence suggested that the abnormal expression of some specific miRNAs are closely related to tumorigenesis and tumor development, and some miRNAs also act as tumor suppressors or oncogenes depending on the characteristic of their target genes (24-26). 
Therefore, further investigation of the expression and roles of miRNAs in GBM is important to develop novel and efficient therapeutic targets for patients with GBM.

The expression and roles of miR-543 have been reported in numerous types of human cancers (27-29). However, miR-543 had not been well studied in GBM. In our present study, we investigated the miR-543 expression pattern in GBM, the effects of miR-543 on GBM cells and the involved molecular mechanism.

\section{Materials and methods}

Tissue sample and cell lines. This study was approved by the Ethical Committee of Shenzhen Second People's Hospital. All patients enrolled in this study gave written informed consent. A total of 19 cases of GBM tissues (age range, 31-69 years; median age, 45; twelve males and seven females) and 8 cases of normal brain tissues were obtained from Department of Neurosurgery, Shenzhen Second People's Hospital between February 2013 and May 2015. Normal brain tissues were collected from internal decompression patients treated with surgical operation. None of these patients had any radiotherapy or chemotherapy before surgical resection. All tissue samples were immediately snap-frozen and stored at $-80^{\circ} \mathrm{C}$ until further use.

Cell lines and transfection. Four GBM cell lines U87, U251, LN229, and T98 were obtained from the Type Culture Collection of the Chinese Academy of Sciences (Shanghai, China), and maintained in Dulbecco's modified Eagle's medium (DMEM) containing 10\% fetal bovine serum (FBS) (both from Invitrogen, Carlsbad, CA, USA), $100 \mathrm{U} / \mathrm{ml}$ penicillin and $100 \mathrm{mg} / \mathrm{ml}$ streptomycin (Invitrogen). Normal human astrocytes (NHAs) purchased from ScienCell Research Laboratories (Carlsbad, CA, USA) were cultured in astrocyte medium (ScienCell Research Laboratories). All cells were grown at $37^{\circ} \mathrm{C}$ in a fully humidified atmosphere containing $5 \% \mathrm{CO}_{2}$ in air.

MiR-543 mimics and miRNA mimics negative control (NC) were obtained from Shanghai GenePharma Co., Ltd. (Shanghai, China). A disintegrin and metalloproteinase 9 (ADAM9) overexpressed plasmid (pcDNA3.1-ADAM9) and blank plasmid (pcDNA3.1) were synthesized by Chinese Academy of Sciences (Changchun, China). Cell transfeciton was performed by using Lipofectamine 2000 (Invitrogen), according to the manufacturer's instructions.

$R N A$ isolation and reverse transcription-quantitative polymerase chain reaction ( $R T-q P C R)$. Total RNA was extracted from tissues or cells using TRIzol (Invitrogen) according to the manufacturer's protocol. To examine miR-543 expression levels, total RNA was transcribed by TaqMan MicroRNA Reverse Transcription kit (Applied Biosystems, Carlsbad, CA, USA). qPCR was performed using TaqMan MicroRNA PCR kit on an ABI Prism 7500 Sequence Detection system (both from Applied Biosystems). To quantify mRNA expression of ADAM9, cDNA was synthesized using a First-Strand cDNA Synthesis kit (Invitrogen) and followed by qPCR with SYBR Premix Ex Taq ${ }^{\mathrm{TM}}$ (Takara Biotechnology Co., Ltd., Dalian, China). U6 and GAPDH was used as internal standard to normalize the expression of miR-543 and ADAM9 mRNA, respectively. The primers were designed as follows: miR-543 forward, 5'-CCAGCTACACTGGGCAGCAGCAATTCA TGTTT-3' and reverse, 5'-CTCAACTGGTGTCGTGGA-3'; U6 forward, 5'-CTCGCTTCGGCAGCACATATACT-3' and reverse, 5'-ACGCTTCACGAATTTGCGTGTC-3'; ADAM9 forward, 5'-GCTAGT TGGACTGGAGATTTGG-3' and reverse, 5'-TTATTACCACAGGAGGGAGCAC-3'; GAPDH forward, 5'-CATCACCATCTTCCAGGAGCG-3' and reverse, 5'-TGACCTTGCCCACAGCCTTG-3'. Relative expression was calculated using the $2^{-\Delta \Delta \mathrm{Ct}}$ method (30).

Cell Counting Kit-8 (CCK-8) assay. CCK-8 assays (Dojindo Molecular Technologies, Kumamoto, Japan) were carried out to assess the cell proliferation. Briefly, transfected cells were trypsinized, collected and seeded into 96-well plate at a density of 3,000 cells/well. Cells were then incubated at $37^{\circ} \mathrm{C}$ with $5 \%$ $\mathrm{CO}_{2}$, and CCK- 8 assay was performed on days $0,1,2$, and 3 . Ten microliters of CCK-8 were added to each well. The plates were incubated at $37^{\circ} \mathrm{C}$ for another $4 \mathrm{~h}$. The absorbance was determined with a microplate reader (Bio-Rad, Richmond, CA, USA) at the wavelength of $450 \mathrm{~nm}$. Each sample was performed in triplicate and repeated three times.

Transwell invasion assay. The invasion assay was performed using Transwell chambers (8 mm pores; Costar, Corning, NY, USA) pre-coated with Matrigel (BD Biosciences, San Jose, CA, USA).

Following transfection for $48 \mathrm{~h}, 5 \times 10^{4}$ cells were added into the top of the Transwell chambers in FBS-free DMEM, and DMEM containing $20 \%$ FBS was added to the lower chambers. The cells were incubated at $37^{\circ} \mathrm{C}$ with $5 \% \mathrm{CO}_{2}$ for $24 \mathrm{~h}$. Cells that did not invade through the pores were removed from the top chambers with a cotton swab while the invasive cells were fixed with $100 \%$ methanol and stained with $0.1 \%$ crystal violet. The number of invasive cells was counted in five random visual fields under an inverted microscope (Olympus Corporation, Tokyo, Japan) and images were captured under x200 magnification.

Flow cytometry analysis. Transfected cells were collected at $48 \mathrm{~h}$ post-transfection, washed with ice-cold PBS and then fixed in $80 \%$ ice-cold ethanol. Subsequently, cells were re-suspended in cold PBS to a concentration of $1 \times 10^{4}$ cells. Annexin V-FITC apoptosis detection kit (Invitrogen) was used to examine cell apoptosis, as described by the manufacturer. The cell apoptotic rates of the transfected cells were determined using a flow cytometry (Beckman Coulter Inc., Brea, CA, USA).

Bioinformatics analysis. The candidate targets of miR-543 predicted by computer-aided algorithms were obtained from TargetScan (www.targetscan.org/) and miRanda (www.microrna.org).

Luciferase reporter assay. Luciferase reporter plasmids, psiCHECK2-ADAM9-3'-UTR-wild type (Wt) and psiCHE CK2-ADAM9-3'-UTR-mutant (Mut), were synthesized and purchased from GenePharma. For luciferase reporter assay,cells were co-transfected with the psiCHECK2-ADAM9-3'-UTR-Wt 
or psiCHECK2-ADAM9-3'-UTR-Mut, and miR-543 mimics or NC using Lipofectamine 2000, following to the manufacturer's instructions. After co-transfection for $48 \mathrm{~h}$, the Dual-Luciferase Reporter Assay system (Promega, Manheim, Germany) was used to measure the activities of Renilla luciferase and firefly luciferase, according to the manufacturer's protocol. Renilla luciferase activity was normalized to firefly luciferase activity.

Western blot analysis. Total protein was extracted with RIPA lysis buffer (Beyotime Institute of Biotechnology, Haimen, China) supplemented with protease inhibitor cocktail (Roche Applied Science, Indianapolis, IN, USA), and the protein concentration was measured using the bicinchoninic acid protocol (Pierce, Rockford, IL, USA). Equal amounts of protein was loaded onto $10 \%$ sodium dodecyl sulphate-polyacrylamide gel and then transferred to polyvinylidene difluoride membranes (Millipore, Billerica, MA, USA). Subsequently, the membrane was blocked with 5\% skimmed milk in TBS $/ 0.1 \%$ Tween (TBST) at room temperature for $1 \mathrm{~h}$, and incubated with primary antibodies overnight at $4^{\circ} \mathrm{C}$. The primary antibodies used in this study were mouse anti-human monoclonal ADAM9 antibody (1:1,000 dilution; sc-377233; Santa Cruz Biotechnology, Santa Cruz CA, USA) and mouse anti-human monoclonal GAPDH antibody $(1: 1,000$ dilution; ab125247; Abcam, Cambridge, UK), at $4^{\circ} \mathrm{C}$ overnight. Following a $2 \mathrm{~h}$ incubation with a goat-anti-mouse horseradish peroxidase (HRP)-conjugated secondary antibody (1:5,000 dilution; sc-2005; Santa Cruz Biotechnology), the protein bands were visualized using the enhanced chemiluminescence kit (Millipore, Billerica, MA, USA). GAPDH was used as a loading control.

Statistical analysis. Data were expressed as mean \pm SD. Statistical analyses were performed using Mann-Whitney's U test or one-way ANOVA with SPSS 16.0 software (SPSS, Chicago, IL, USA). P $<0.05$ was considered to be statistically significant.

\section{Results}

Reduced miR-543 expression levels in GBM tissues and cell lines. The expression of miR-543 in 19 cases of GBM tissues and 8 cases of normal brain tissues was investigated using RT-qPCR to explore the potential roles of miR-543 in GBM. As shown in Fig. 1A, miR-543 expression level was significantly decreased in GBM tissue compared with that in normal brain tissues $(\mathrm{P}<0.05)$. The expression of miR-543 in four GBM cell lines (U87, U251, LN229, T98) and NHAs was determined to confirm the reduced miR-543 expression level in GBM tissues. miR-543 expression level was lower in GBM cell lines than that in NHAs (Fig. 1B, $\mathrm{P}<0.05$ ). These results suggested that miR-543 was downregulated in GBM and may act as a tumor suppressor.

MiR-543 inhibits the proliferation and invasion, as well as enhances the apoptosis of GBM cells. To ascertain the functional effect of miR-543 on GBM, we transfected the two lowest miR-543 expressing cell lines, U87 and U251, with miR-543 mimic to increase its endogenous expression levels.
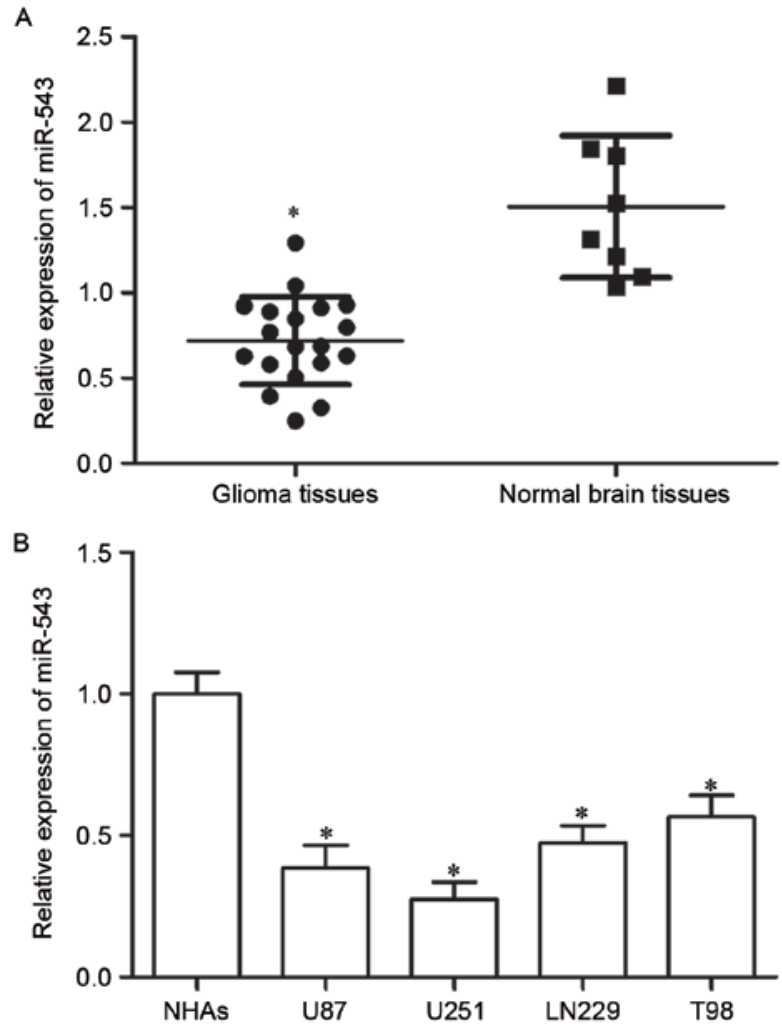

Figure 1. Analysis of miR-543 expression in GBM tissues and cell lines (A) Relative miR-543 expression in 19 cases of GBM tissues and 8 cases of normal brain tissues was examined by RT-qPCR. "P $<0.05$ compared with normal brain tissues. (B) Expression of miR-543 in four GBM cell lines (U87, U251, LN229, T98) and NHAs was determined by RT-qPCR. ${ }^{*} \mathrm{P}<0.05$ compared with normal brain tissues. NHAs, normal human astrocytes; GBM, glioblastoma.

After 48-h transfection, RT-qPCR confirmed that miR-543 was markedly upregulated in U87 and U251 cells transfected with miR-543 mimics (Fig. $2 \mathrm{~A}$ and $\mathrm{B}, \mathrm{P}<0.05$ ). CCK-8 assays were utilized to investigate the effect of miR-543 on GBM cell proliferation. As shown in Fig. 2C and D, U87 and U251 cells with high miR-543 expression levels exhibited a significant inhibition of proliferation compared with the cells with NC. Then, the effect of miR-543 on GBM cell invasion was analysed using Transwell invasion assay. Results showed that the upregulation of miR-543 suppresses the U87 and U251 cell invasion abilities compared with cells transfected with NC (Fig. 2E and F, P<0.05). Flow cytometry analysis was performed in U87 and U251 cells transfected with miR-543 mimics or NC to examine whether miR-543 overexpression has a positive effect on GBM cell apoptosis. We detected a clear apoptosis activation in miR-543 mimics-transfected U87 and $\mathrm{U} 251$ cells (Fig. $2 \mathrm{G}$ and $\mathrm{H}, \mathrm{P}<0.05$ ). These results indicated that miR-543 exerts tumor-suppressing roles in GBM cells.

ADAM9 is a direct target of miR-543 in GBM. Bioinformatics analysis was conducted to identify the candidate target genes of miR-543 and to investigate the underlying mechanism by which miR-543 regulates GBM cell proliferation, invasion and apoptosis. Among these predicted targets, ADAM9, which is an important regulator for cancer formation and progression (31-33), attracted attention and was selected for 
A

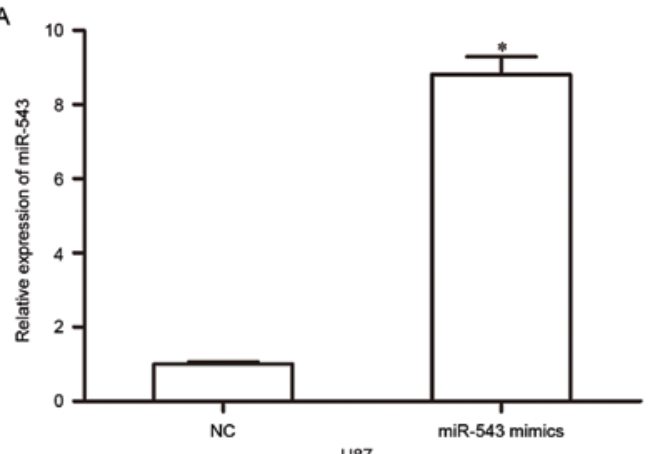

U87

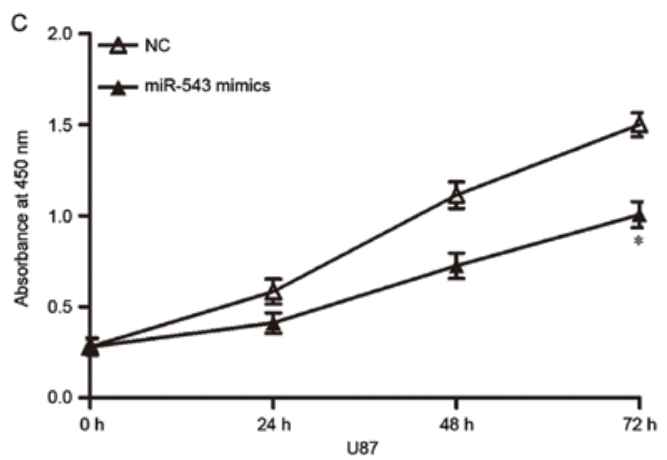

E
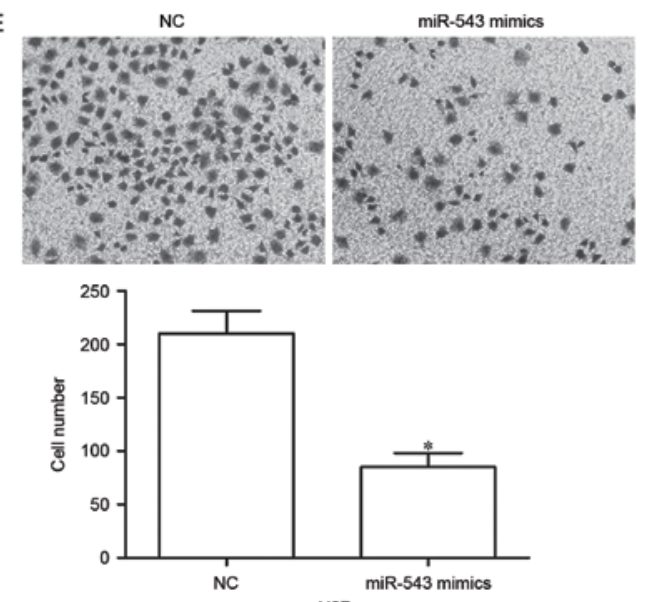

U87
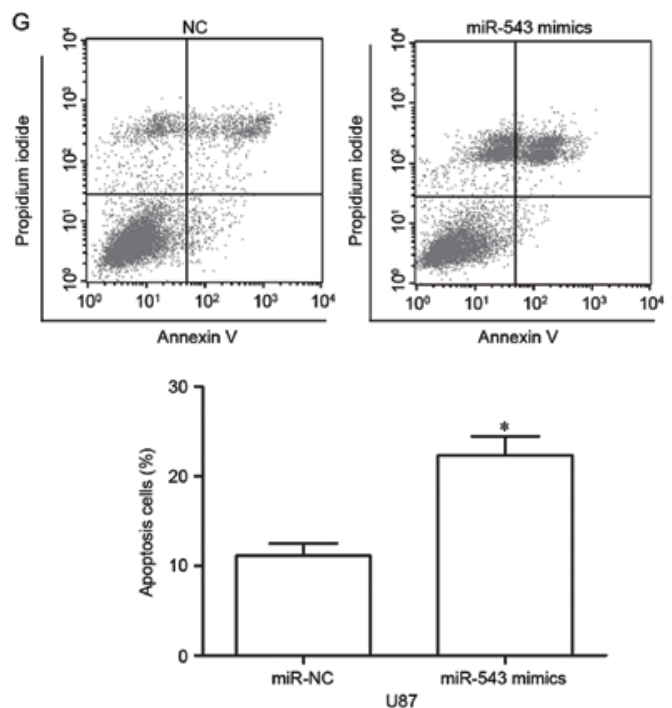

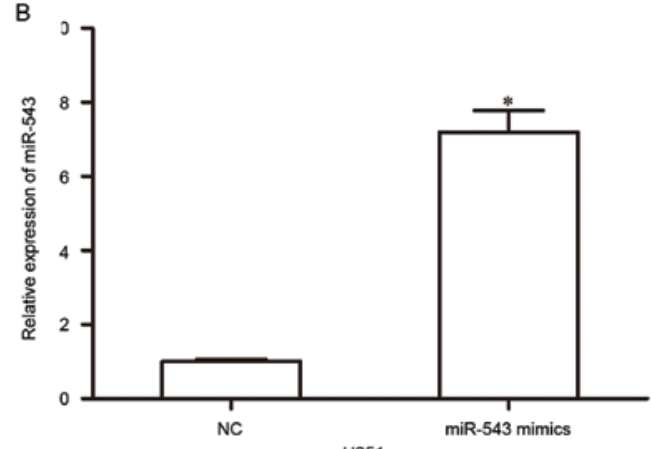

U251

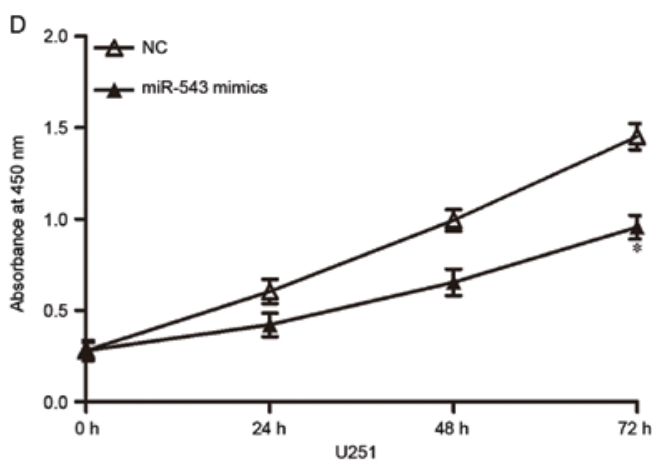

F
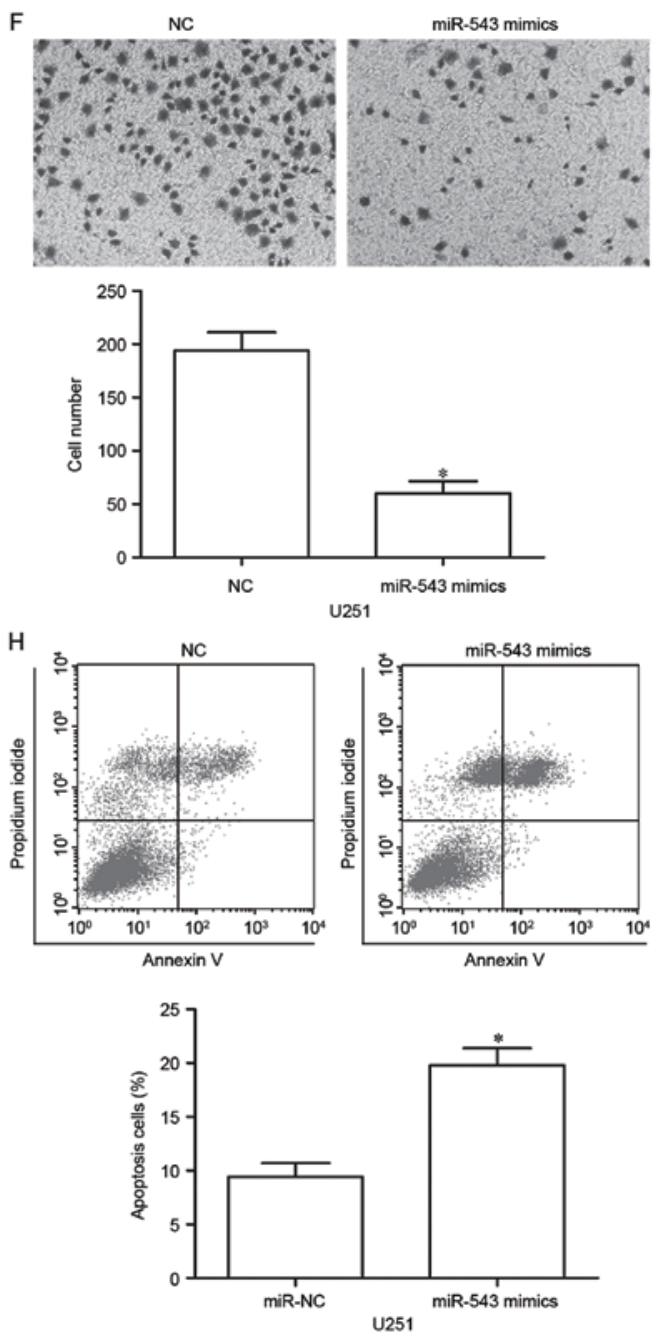

Figure 2. Restoration expression of miR-543 inhibited tumour progression in GBM cells. (A and B) Expression levels of miR-543 in U87 and U251 cells transfected with miR-543 mimics or NC were assessed using RT-qPCR. "P<0.05 compared with NC. (C and D) Relative cell proliferation in U87 and U251 cells was examined after the cells transfected with miR-543 mimics or NC using CCK-8 assays. "P<0.05 compared with NC. (E and F) Effect of miR-543 on cell invasion was evaluated by using Transwell invasion assay. ${ }^{*} \mathrm{P}<0.05$ compared with NC. (G and H) Apoptotic rate of U87 and U251 cells transfected with miR-543 mimics or NC were measured using flow cytometry analysis and compared with that of the cells involving NC. "P<0.05 compared with NC. GBM, glioblastoma; CCK-8, Cell Counting Kit-8. 
A
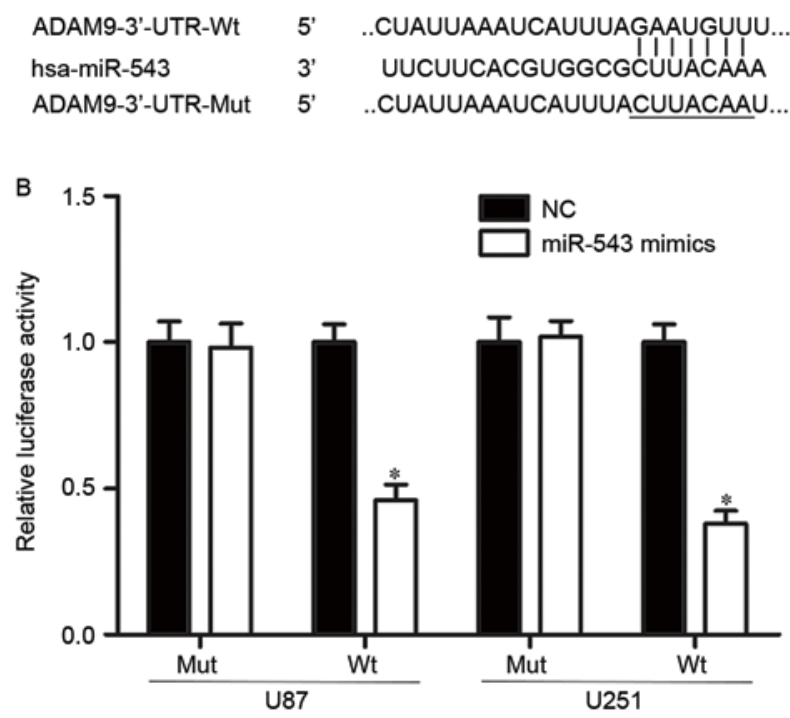

C

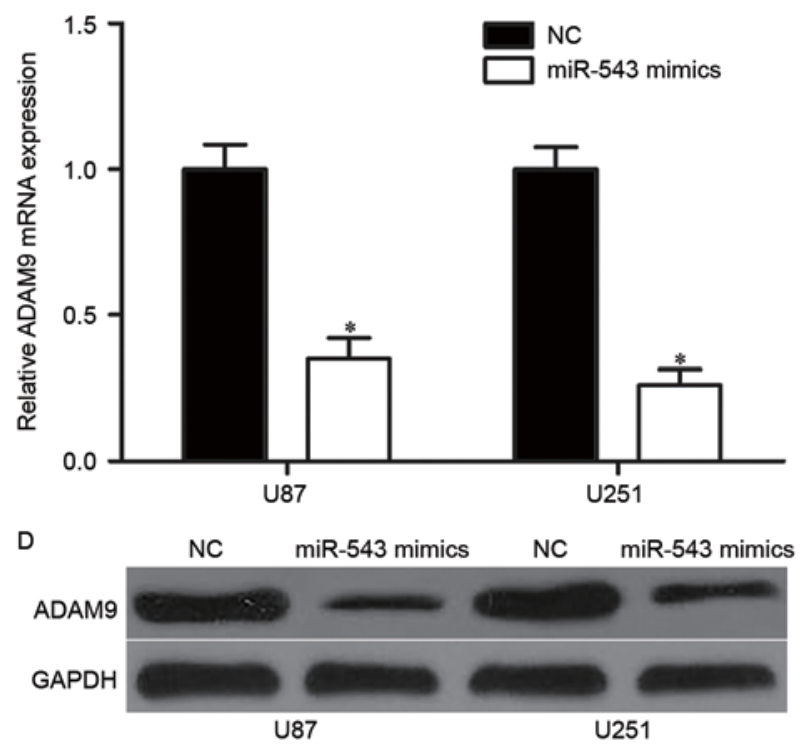

Figure 3. ADAM9 is a target of miR-543 in GBM. (A) Putative seed-matching sites or mutant sites between miR-543 and the 3'-UTR of ADAM9. (B) Luciferase reporter assays in U87 and U251 cells after co-transfection of cells with psiCHECK2-ADAM9-3'-UTR-Wt or psiCHECK2-ADAM9-3'-UTR-Mut and miR-543 mimics or NC. * $\mathrm{P}<0.05$ compared with NC. (C and D) Effect of miR-543 overexpression on endogenous ADAM9 nRNA and protein was determined by RT-qPCR and western blot analysis, respectively. "P<0.05 compared with NC. ADAM9, a disintegrin and metalloproteinase 9; GBM, glioblastoma; 3'-UTR, 3'-untranslated region.

further confirmation (Fig. 3A). Luciferase reporter assay was performed on U87 and U251 cells to validate interaction between miR-543 and the predicted binding site of ADAM9. The overexpression of miR-543 decreased wild-type (Fig. 3B, $\mathrm{P}<0.05$ ) but not mutant ADAM9 reporter activity, suggesting that miR-543 specifically targeted the 3'UTR of ADAM9. To confirm whether ADAM9 are regulated by miR-543, the mRNA and protein expression of ADAM9 were examined in U87 and U251 cells transfected with miR-543 mimics or NC. Results showed that restoration expression of miR-543 reduced the ADAM9 expression levels in U87 and U251 cells at both mRNA and protein levels (Fig. $3 \mathrm{C}$ and $\mathrm{D}, \mathrm{P}<0.05$ ). These results suggested that ADAM9 is a direct target of miR-543 in GBM.

ADAM9 is upregulated in GBM tissues and inversely correlates with miR-543 levels. Further experiments were utilized to detect the ADAM9 expression in 19 cases of GBM tissues and eight cases of normal brain tissues. RT-qPCR and Western blot results revealed that ADAM9 mRNA and protein expression levels were higher in GBM tissues relative to that in normal brain tissues (Fig. 4A and B, P<0.05). Spearman's correlation analysis was then performed to explore the relationship between miR-543 and ADAM9 mRNA expression in GBM tissues. As shown in Fig. 4C, ADAM9 mRNA was inversely correlated with miR-543 levels in GBM tissues (Fig. 4C, $r=-0.6685$, $\mathrm{P}=0.0018$ ), suggesting that the downregulation of miR-543 may be an important cause for the upregulation of ADAM9 in GBM.

Upregulation of ADAM9 reverses the effects of miR-543 on GBM cell proliferation, invasion and apoptosis. Rescue experiments were performed in U87 and U251 cells to further explore the role of ADAM9 on the biological roles induced by miR-543 in GBM. Then, western blot analysis was used to measure the ADAM9 expression in U87 and U251 cells transfected with pcDNA3.1-ADAM9 or pcDNA3.1. Our data showed that ADAM9 protein was significantly upregulated in U87 and U251 cells after transfection with pcDNA3.1-ADAM9 (Fig. 5A, $\mathrm{P}<0.05$ ). Functional rescue results showed that the reintroduction of ADAM9 reversed the effects of miR-543 overexpression on GBM cell proliferation (Fig. 5B and C, P<0.05), invasion (Fig. 5D and E, $\mathrm{P}<0.05$ ) and apoptosis (Fig. 5F and $\mathrm{G}, \mathrm{P}<0.05$ ). These results indicated that miR-543 inhibits GBM cell proliferation and invasion, as well as promotes apoptosis by targeting ADAM9.

\section{Discussion}

Recently, accumulated studies demonstrated that abnormally expressed miRNAs may act as oncogenes or tumor suppressor genes and is important on the formation and progression of various human cancers, including GBM (34-36). Therefore, investigation on the expression and roles of miRNAs may have potential tumor diagnostic and prognostic values, as well as therapeutic values for patients with GBM (37). In our current study, miR-543 was lowly expressed in GBM tissues and cell lines. The resumption expression of miR-543 inhibited the proliferation and invasion, as well as enhanced the apoptosis of GBM cells. In addition, ADAM9 was identified as a novel target of miR-543. Therefore, miR-543 played tumor suppressing roles through the downregulation of ADAM9 in GBM.

miR-543 was aberrantly expressed in numerous types of human cancers. For example, in colorectal cancer, miR-543 was downregulated in tumor tissues and cell lines. The low miR-543 expression level was inversely correlated with the metastatic status of patients with colorectal cancer and the metastatic 

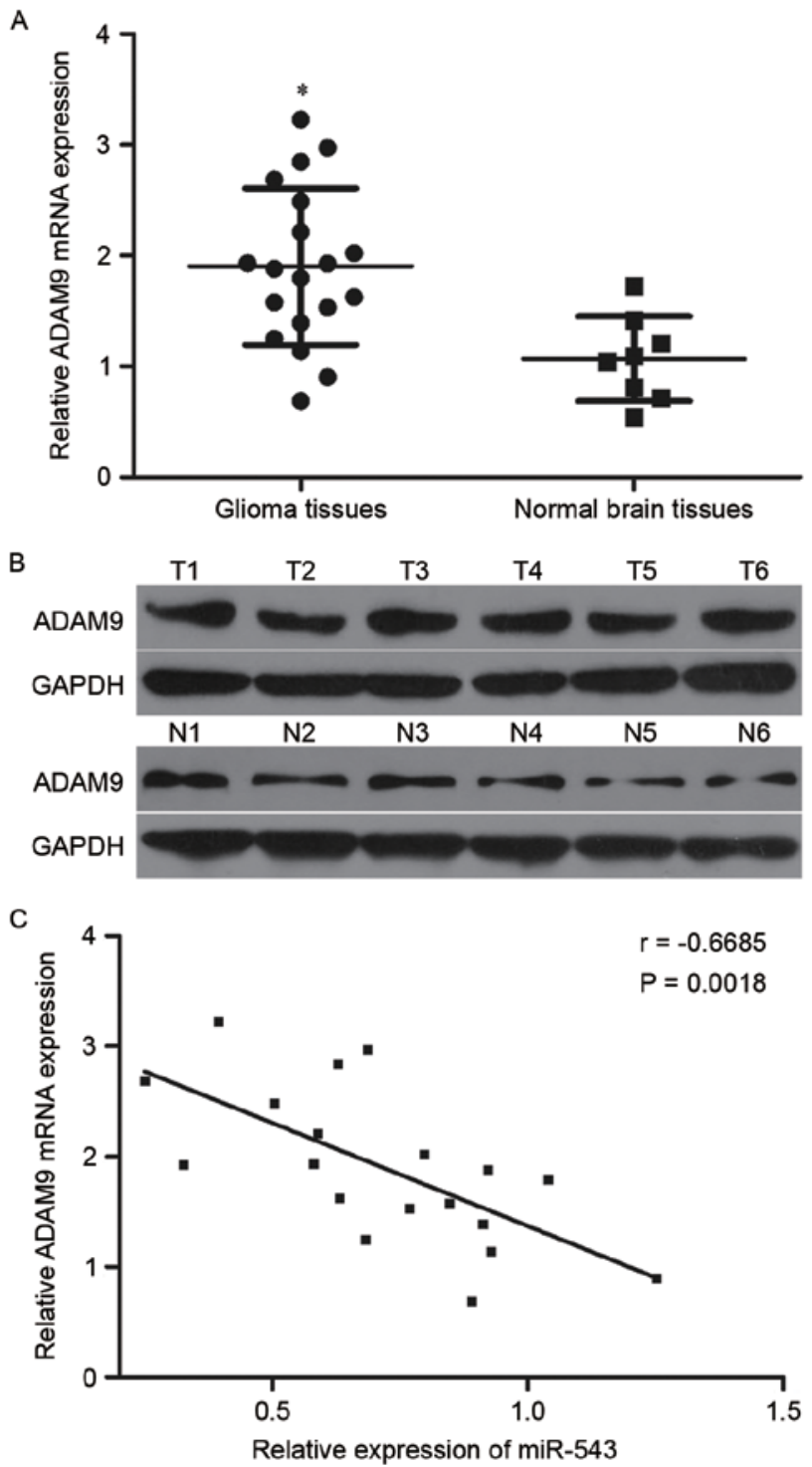

Figure 4. ADAM9 expression was upregulated and negatively correlated with miR-543 expression in GBM tissues. (A and B) RT-qPCR and western blot analysis were performed to detect ADAM9 mRNA and protein expression in GBM tissues and normal brain tissues. " $\mathrm{P}<0.05$ compared with normal brain tissues. (C) Negative relationship between ADAM9 mRNA and miR-543 expression was analysed by Spearman's correlation analysis in GBM tissues. ADAM9, a disintegrin and metalloproteinase 9; GBM, glioblastoma.

potential of colorectal cancer cell lines (27). miR-543 was lowly expressed in endometrial cancer (28). However, contrary to these results, miR-543 was upregulated in gefitinib-resistant non-small cell lung cancer (29), hepatocellular carcinoma (38), gastric cancer (39) and osteosarcoma (40). These conflicting findings indicated that the expression pattern of miR-543 in human cancer has tissue specificity.

miR-543 has been demonstrated to be a tumor suppressor. Fan et al reported that the enforced expression of miR-543 suppressed the proliferation and metastasis of colorectal cancer cells both in vitro and in vivo (27). Bing et al revealed that miR-543 re-expression inhibited cell monolayer proliferation, anchorage-independent growth, migration and invasion of endometrial cancer (28). However, in non-small cell lung cancer, Bi et al found that miR-543 acts as an oncogene by attenuating tumor cell proliferation and invasion (29). Yu et al demonstrated that the upregulation of miR-543 enhanced the cell growth and motility of hepatocellular carcinoma (38). Li et al showed that the ectopic expression of miR-543 promoted the cell proliferation and cell cycle progression of gastric cancer (39). Besides, the restoration expression of miR-543 promoted the osteosarcoma cell growth in vitro and in vivo (40).

These studies may appear contradictory, because miR-543 acted as an oncogene in certain types of cancer and a tumor suppressor in others. These contradictory results may be explained by the imperfect complementarity of the interactions between miRNAs and their target genes (41).

Identification of cancer-specific miRNAs and their target genes is pivotal for understanding their roles in tumorigenesis and tumor development $(42,43)$. Several target genes of miR-543 have been identified, such as KRAS, MTA1 and HMGA2 in colorectal cancer (27), FAK and TWIST1 in endometrial cancer (28), PTEN in lung cancer (29), PAQR3 in hepatocellular carcinoma (38), SIRT1 in gastric cancer (39) and PRMT9 in osteosarcoma (40). Herein, we focused on the mechanisms of miR-543 in regulating cell proliferation, invasion and apoptosis of GBM cells. Bioinformatics analysis was performed to search candidate targets of miR-543, among which ADAM9 was predicated as a potential target of miR-543. Luciferase reporter assay indicated that miR-543 directly targeted the 3'-UTR of ADAM9. Following, we confirmed that ADAM9 mRNA and protein levels were negatively regulated by miR-543 in GBM cells. Additionally, ADAM9 was upregulated in GBM tissues and inversely correlated with miR-543 expression level. Besides, rescue experiments demonstrated that upregulation of ADAM9 rescued the effects induced by miR-543 overexpression on GBM cells. These results suggest that miR-543 can directly and negatively regulate ADAM9 expression by binding to the 3'-UTR of ADAM9.

ADAMs are members of the metzincin superfamily of matrix metalloproteinases (MMP) (44). To date, 21 functional ADAMs have been described in humans and 40 in different organisms (45). ADAMs are involved in many different biological functions, including fertilization, adhesion, migration and proteolysis $(44,46)$. ADAM9 is a membrane-anchored metalloproteinase and is one of the first ADAM proteins to be identified and characterized. It consists of an N-terminal prodomain followed by a metalloprotease domain, a disintegrin domain and cysteine-rich region, an epidermal growth factor repeat, a transmembrane domain and a cytoplasmic tail with potential SH3 ligand domains $(47,48)$. Previous studies reported that ADAM9 was upregulated in several types of human cancers, such as renal cell cancer (31), non-small cell lung cancer (49), prostate cancer (50), hepatocellular carcinoma (32), colon cancer (33) and pancreatic cancer (51). In glioma, ADAM9 was highly expressed in tumor tissues. In addition, high expression levels of ADAM9 were significantly correlated with poor prognosis in lower-grade glioma. Furthermore, multivariate analysis indicated ADAM9 expression as an independent marker of poor survival (52). Functional assays revealed that ADAM9 plays important roles in glioma growth and metastasis $(19,53)$. These studies all indicated that ADAM9 may be a novel and promising target for therapeutic intervention in patients with glioma. 


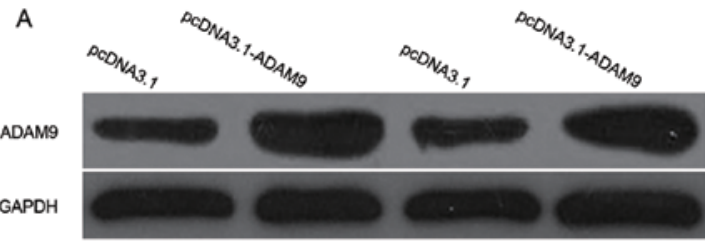

U87

B

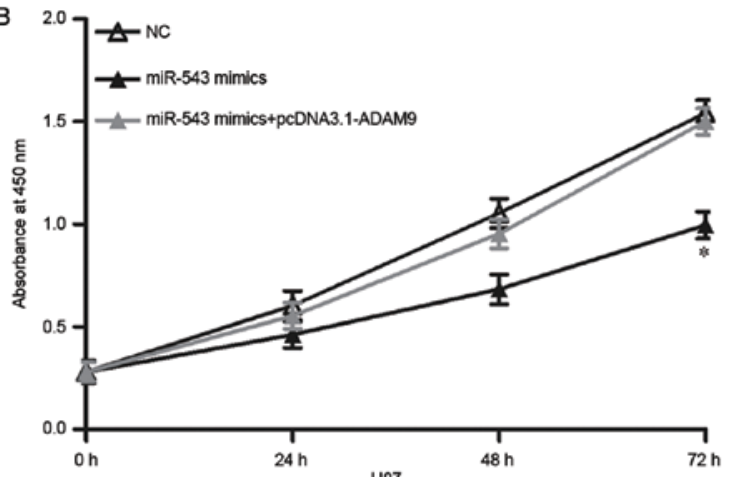

D
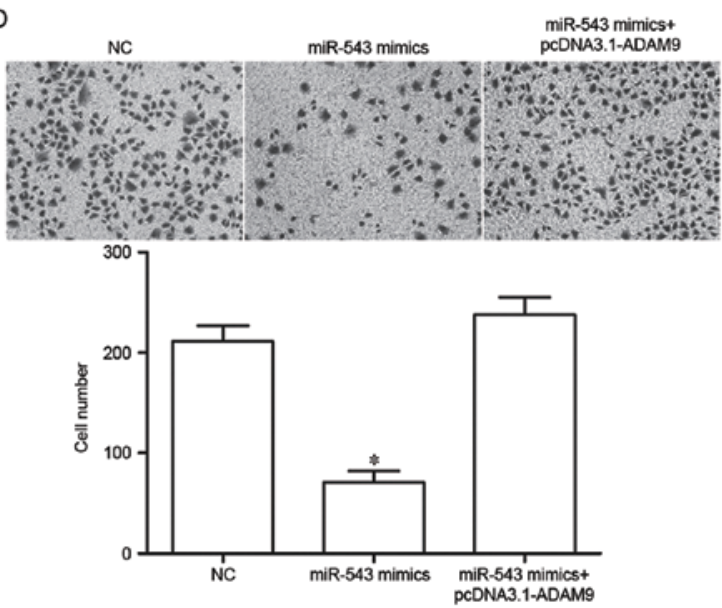

U87
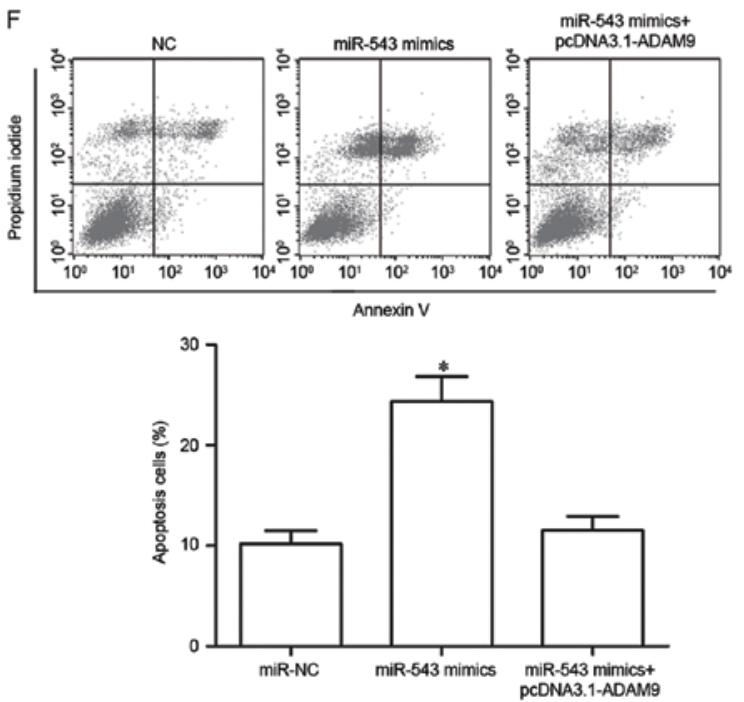

U87
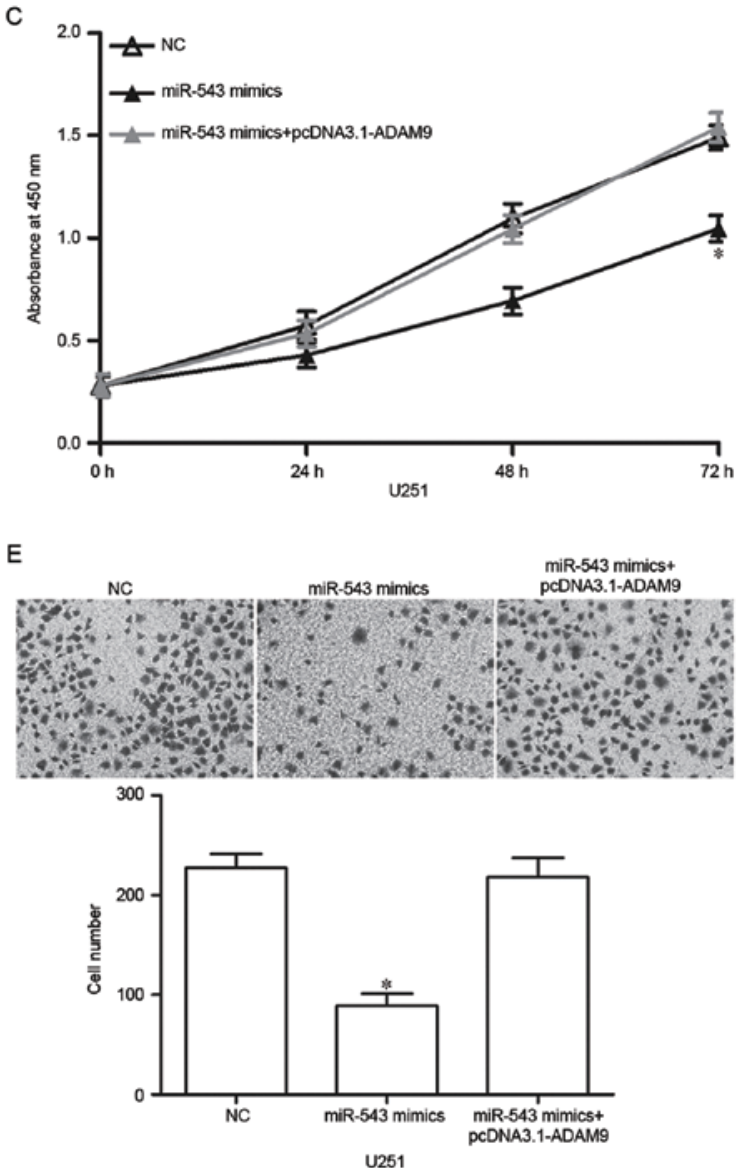

G
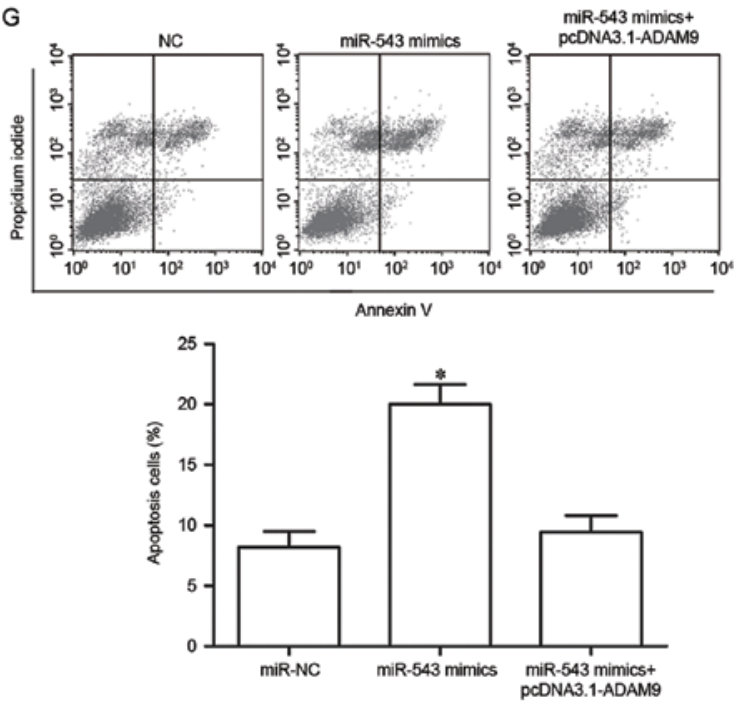

U251

Figure 5. Overexpression of ADAM9 reversed miR-543-induced tumour suppressive effects on GBM cell behaviours. (A) Western blot analysis showed that ADAM9 protein expression level was increased in U87 and U251 cells transfected with pcDNA3.1-ADAM9. * $<0.05$ compared with pcDNA3.1. (B and C) CCK-8 assay was used to detect proliferation in U87 and U251 cells treated with NC, miR-543 mimics or miR-543 mimics+pcDNA3.1-ADAM9. ${ }^{*} \mathrm{P}<0.05$ compared with NC and miR-543 mimics+pcDNA3.1-ADAM9. (D and E) Transwell invasion assay of U87 and U251 cells transfected with NC, miR-543 mimics or miR-543 mimics+pcDNA3.1-ADAM9. "P<0.05 compared with NC and miR-543 mimics+pcDNA3.1-ADAM9. (F and G) Flow cytometry analysis of U87 and U251 cells transfected with NC, miR-543 mimics or miR-543 mimics+pcDNA3.1-ADAM9. "P<0.05 compared with NC and miR-543 mimics+pcDNA3.1-ADAM9. ADAM9, a disintegrin and metalloproteinase 9; GBM, glioblastoma; CCK-8, Cell Counting Kit-8. 
In conclusion, miR-543 was downregulated in GBM and played an important role in regulating GBM cell proliferation, invasion and apoptosis. Moreover, ADAM9 is the target gene of miR-543. These results suggested that miR-543/ADAM9 pathway may be investigated as an important strategy for the treatment of patients with GBM.

\section{References}

1. Jungk C, Chatziaslanidou D, Ahmadi R, Capper D, Bermejo JL, Exner J, von Deimling A, Herold-Mende C and Unterberg A: Chemotherapy with BCNU in recurrent glioma: Analysis of clinical outcome and side effects in chemotherapy-naive patients. BMC Cancer 16: 81, 2016.

2. Wu CX, Lin GS, Lin ZX, Zhang JD, Chen L, Liu SY, Tang WL, Qiu XX and Zhou CF: Peritumoral edema on magnetic resonance imaging predicts a poor clinical outcome in malignant glioma. Oncol Lett 10: 2769-2776, 2015.

3. Hatanpaa KJ, Burma S, Zhao D and Habib AA: Epidermal growth factor receptor in glioma: Signal transduction, neuropathology, imaging, and radioresistance. Neoplasia 12: 675-684, 2010.

4. Cohen AL, Holmen SL and Colman H: IDH1 and IDH2 mutations in gliomas. Curr Neurol Neurosci Rep 13: 345, 2013.

5. Endersby R and Baker SJ: PTEN signaling in brain: Neuropathology and tumorigenesis. Oncogene 27: 5416-5430, 2008.

6. Komori T, Sasaki H and Yoshida K: Revised WHO classification of tumours of the central nervous system: Summary of the revision and perspective. No Shinkei Geka 44: 625-635, 2016 (In Japanese).

7. Minniti G, Muni R, Lanzetta G, Marchetti P and Enrici RM: Chemotherapy for glioblastoma: Current treatment and future perspectives for cytotoxic and targeted agents. Anticancer Res 29: 5171-5184, 2009.

8. Schwartzbaum JA, Fisher JL, Aldape KD and Wrensch M: Epidemiology and molecular pathology of glioma. Nat Clin Pract Neurol 2: 494-503, 2006.

9. Yan Y, Peng Y, Ou Y and Jiang Y: MicroRNA-610 is downregulated in glioma cells, and inhibits proliferation and motility by directly targeting MDM2. Mol Med Rep 14: 2657-2664, 2016.

10. Wen PY and Kesari S: Malignant gliomas in adults. N Engl J Med 359: 492-507, 2008.

11. Furnari FB, Fenton T, Bachoo RM, Mukasa A, Stommel JM, Stegh A, Hahn WC, Ligon KL, Louis DN, Brennan C, et al Malignant astrocytic glioma: Genetics, biology, and paths to treatment. Genes Dev 21: 2683-2710, 2007.

12. Pollack IF: Neuro-oncology: Therapeutic benefits of reirradiation for recurrent brain tumors. Nat Rev Neurol 6: 533-535, 2010.

13. Ameres SL and Zamore PD: Diversifying microRNA sequence and function. Nat Rev Mol Cell Biol 14: 475-488, 2013.

14. Bartel DP: MicroRNAs: Genomics, biogenesis, mechanism, and function. Cell 116: 281-297, 2004.

15. Osaki M, Okada F and Ochiya T: miRNA therapy targeting cancer stem cells: A new paradigm for cancer treatment and prevention of tumor recurrence. Ther Deliv 6: 323-337, 2015.

16. Hwang HW and Mendell JT: MicroRNAs in cell proliferation, cell death, and tumorigenesis. Br J Cancer 96 (Suppl): R40-R44, 2007.

17. Calin GA and Croce CM: MicroRNA signatures in human cancers. Nat Rev Cancer 6: 857-866, 2006.

18. Bushati N and Cohen SM: MicroRNA functions. Annu Rev Cell Dev Biol 23: 175-205, 2007.

19. Liu X, Wang S, Yuan A, Yuan X and Liu B: MicroRNA-140 represses glioma growth and metastasis by directly targeting ADAM9. Oncol Rep 36: 2329-2338, 2016.

20. Guan H, Liu C, Fang F, Huang Y, Tao T, Ling Z, You Z, Han X, Chen S, Xu B and Chen M: MicroRNA-744 promotes prostate cancer progression through aberrantly activating $\mathrm{Wnt} / \beta$-catenin signaling. Oncotarget 8: 14693-14707, 2017.

21. Qi M, Liu D and Zhang S: MicroRNA-21 contributes to the discrimination of chemoresistance in metastatic gastric cancer. Cancer Biomark 18: 451-458, 2017.

22. An N, Luo X, Zhang M and Yu R: MicroRNA-376b promotes breast cancer metastasis by targeting Hoxd10 directly. Exp Ther Med 13: 79-84, 2017.

23. Zhang Y, An J, Lv W, Lou T, Liu Y and Kang W: miRNA-129-5p suppresses cell proliferation and invasion in lung cancer by targeting microspherule protein 1 , E-cadherin and vimentin. Oncol Lett 12: 5163-5169, 2016.
24. Lu J, Getz G, Miska EA, Alvarez-Saavedra E, Lamb J, Peck D, Sweet-Cordero A, Ebert BL, Mak RH, Ferrando AA, et al: MicroRNA expression profiles classify human cancers. Nature 435: 834-838, 2005.

25. Peng Z, Wu T, Li Y, Xu Z, Zhang S, Liu B, Chen Q and Tian D: MicroRNA-370-3p inhibits human glioma cell proliferation and induces cell cycle arrest by directly targeting $\beta$-catenin. Brain Res 1644: 53-61, 2016.

26. Hao Y, Zhang S, Sun S, Zhu J and Xiao Y: miR-595 targeting regulation of SOX7 expression promoted cell proliferation of human glioblastoma. Biomed Pharmacother 80: 121-126, 2016.

27. Fan C, Lin Y, Mao Y,Huang Z, Liu AY, Ma H, Yu D, Maitikabili A, Xiao H, Zhang C, et al: MicroRNA-543 suppresses colorectal cancer growth and metastasis by targeting KRAS, MTA1 and HMGA2. Oncotarget 7: 21825-21839, 2016.

28. Bing L, Hong C, Li-Xin S and Wei G: MicroRNA-543 suppresses endometrial cancer oncogenicity via targeting FAK and TWIST1 expression. Arch Gynecol Obstet 290: 533-541, 2014.

29. Bi M, Chen W, Yu H, Wang J, Ding F, Tang DJ and Tang C: miR-543 is up-regulated in gefitinib-resistant non-small cell lung cancer and promotes cell proliferation and invasion via phosphatase and tensin homolog. Biochem Biophys Res Commun 480: 369-374, 2016.

30. Livak KJ and Schmittgen TD: Analysis of relative gene expression data using real-time quantitative PCR and the 2(-Delta Delta C(T)) method. Methods 25: 402-408, 2001.

31. Fritzsche FR, Wassermann K, Jung M, Tölle A, Kristiansen I, Lein M, Johannsen M, Dietel M, Jung K and Kristiansen G: ADAM9 is highly expressed in renal cell cancer and is associated with tumour progression. BMC Cancer 8: 179, 2008.

32. Tao K, Qian N, Tang Y, Ti Z, Song W, Cao D and Dou K: Increased expression of a disintegrin and metalloprotease-9 in hepatocellular carcinoma: Implications for tumor progression and prognosis. Jpn J Clin Oncol 40: 645-651, 2010.

33. Li J, Ji Z, Qiao C, Qi Y and Shi W: Overexpression of ADAM9 Promotes Colon Cancer Cells Invasion. J Invest Surg 26: 127-133, 2013.

34. Liu N, Zhang L, Wang Z, Cheng Y, Zhang P, Wang X, Wen W, Yang H, Liu H, Jin W, et al: MicroRNA-101 inhibits proliferation, migration and invasion of human glioblastoma by targeting SOX9. Oncotarget 8: 19244-19254, 2017.

35. Li D, Wang Z, Chen Z, Lin L, Wang Y, Sailike D, Luo K, Du G, Xiang X and Jiafu GD: MicroRNA-106a-5p facilitates human glioblastoma cell proliferation and invasion by targeting adenomatosis polyposis coli protein. Biochem Biophys Res Commun 481: 245-250, 2016.

36. Wang J, Liu H, Tian L, Wang F, Han L, Zhang W and Bai YA: miR-15b Inhibits the progression of glioblastoma cells through targeting insulin-like growth factor receptor 1 . Horm Cancer 8: 49-57, 2017

37. Karsy M, Arslan E and Moy F: Current progress on understanding MicroRNAs in glioblastoma multiforme. Genes Cancer 3: 3-15, 2012.

38. Yu L, Zhou L, Cheng Y, Sun L, Fan J, Liang J, Guo M, Liu N and Zhu L: MicroRNA-543 acts as an oncogene by targeting PAQR3 in hepatocellular carcinoma. Am J Cancer Res 4: 897-906, 2014.

39. Li J, Dong G, Wang B, Gao W and Yang Q: miR-543 promotes gastric cancer cell proliferation by targeting SIRT1. Biochem Biophys Res Commun 469: 15-21, 2016.

40. Zhang H, Guo X, Feng X, Wang T, Hu Z, Que X, Tian Q, Zhu T, Guo G, Huang W and Li X: miRNA-543 promotes osteosarcoma cell proliferation and glycolysis by partially suppressing PRMT9 and stabilizing HIF-1 $\alpha$ protein. Oncotarget 8: 2342-2355, 2017.

41. Yu Z, Ni L, Chen D, Zhang Q, Su Z, Wang Y, Yu W, Wu X, Ye J, Yang $\mathrm{S}$, et al: Identification of miR-7 as an oncogene in renal cell carcinoma. J Mol Histol 44: 669-677, 2013.

42. Song L, Yang J, Duan P, Xu J, Luo X, Luo F, Zhang Z, Hou T, Liu B and Zhou Q: MicroRNA-24 inhibits osteosarcoma cell proliferation both in vitro and in vivo by targeting LPAAT $\beta$. Arch Biochem Biophys 535: 128-135, 2013.

43. Wu X, Zhong D, Gao Q, Zhai W, Ding Z and Wu J: MicroRNA-34a inhibits human osteosarcoma proliferation by downregulating ether à go-go 1 expression. Int J Med Sci 10: 676-682, 2013.

44. Blobel CP: ADAMs: Key components in EGFR signalling and development. Nat Rev Mol Cell Biol 6: 32-43, 2005.

45. Nath D, Slocombe PM, Stephens PE, Warn A, Hutchinson GR, Yamada KM, Docherty AJ and Murphy G: Interaction of metargidin (ADAM-15) with alphavbeta3 and alpha5beta1 integrins on different haemopoietic cells. J Cell Sci 112: 579-587, 1999. 
46. Edwards DR, Handsley MM and Pennington CJ: The ADAM metalloproteinases. Mol Aspects Med 29: 258-289, 2008.

47. Guaiquil V, Swendeman S, Yoshida T, Chavala S, Campochiaro PA and Blobel CP: ADAM9 is involved in pathological retinal neovascularization. Mol Cell Biol 29: 2694-2703, 2009.

48. Duffy MJ, McKiernan E, O'Donovan N and McGowan PM: Role of ADAMs in cancer formation and progression. Clin Cancer Res 15: 1140-1144, 2009.

49. Zhang J, Qi J, Chen N, Fu W, Zhou B and He A: High expression of a disintegrin and metalloproteinase-9 predicts a shortened survival time in completely resected stage I non-small cell lung cancer. Oncol Lett 5: 1461-1466, 2013.

50. Fritzsche FR, Jung M, Tölle A, Wild P, Hartmann A, Wassermann K, Rabien A, Lein M, Dietel M, Pilarsky C, et al: ADAM9 expression is a significant and independent prognostic marker of PSA relapse in prostate cancer. Eur Urol 54: 1097-1106, 2008.
51. Grutzmann R, Lüttges J, Sipos B, Ammerpohl O, Dobrowolski F, Alldinger I, Kersting S, Ockert D, Koch R, Kalthoff H, et al: ADAM9 expression in pancreatic cancer is associated with tumour type and is a prognostic factor in ductal adenocarcinoma. Br J Cancer 90: 1053-1058, 2004.

52. Fan X, Wang Y, Zhang C, Liu L, Yang S, Wang Y, Liu X, Qian Z, Fang S, Qiao H and Jiang T: ADAM9 expression is associate with glioma tumor grade and histological type and acts as a prognostic factor in lower-grade gliomas. Int J Mol Sci 17: pii: E1276, 2016

53. Chen CM, Hsieh YH, Hwang JM, Jan HJ, Hsieh SC, Lin SH and Lai CY: Fisetin suppresses ADAM9 expression and inhibits invasion of glioma cancer cells through increased phosphorylation of ERK1/2. Tumour Biol 36: 3407-3415, 2015. 